\title{
PREDICTING DRIVING DISTRACTION PATERNS IN DIFFERENT ROAD CLASSES USING A SUPPORT VECTOR MACHINE
}

\author{
Samira Ahangari ${ }^{1}$, Mansoureh Jeihani ${ }^{2}$, Md Mahmudur Rahman $^{3}$, Abdollah Dehzangi $^{4}$ \\ 1,2 Department of Transportation and Infrastructure Studies, Morgan State University, Baltimore, \\ MD 21251, USA \\ ${ }^{3,4}$ Department of Computer Science, Morgan State University, Baltimore, MD 21251, USA
}

Received 17 February 2020; accepted 12 October 2020

\begin{abstract}
This study investigates driving behavior under distraction on four different road classes - freeway, urban arterial, rural, and local road in a school zone - using a high-fidelity driving simulator. Some 92 younger participants from a reasonably diverse sociodemographic background drove a realistic midsize network in the Baltimore metropolitan area and were exposed to different distractions. A total of 1,952 simulation runs were conducted. An ANOVA and Tukey Post Hoc analysis showed that distracted driving behavior demonstrates different patterns on various roads. This research developed a support vector machine model that achieved distraction prediction ability among different routes with an accuracy of $94.24 \%$, which to the best of our knowledge, is the best for such a task. The results indicate that driver distraction prediction models probably would be more accurate if developed separately for each road class.
\end{abstract}

Keywords: distracted driving, driving simulator, ANOVA, post hoc tukey, machine learning, support vector machine.

\section{Introduction}

One of the most significant traffic safety problems is driver distraction. According to the National Highway Traffic Safety Administration (NHTSA), $9 \%$ of the 37,133 fatal crashes are attributed to driver distraction, which involved 2,994 distracted drivers in 2017 (NHTSA, 2015). The distraction problem is getting worse due to the increasing use of in-vehicle information systems such as GPS navigation systems, cellphones, and satellite radios (Lee et al., 2001; Strayer et al., 2003). Modern vehicles (Ebnali et al., 2020) are filled with driverassistance technology such as a navigator, multimedia displays, climate control, parking radar, and many more. Although drivers benefit from such modern driving assistance technologies (Ahangari et al., 2018, 2019), it is still critical for drivers to avoid distraction and pay suitable attention to the road.

It was shown previously that wireless devices, conversations with a passenger, and invehicle distraction are the significant sources of incidents (Haigney \& Westerman, 2001). Also, the growing use of mobile phones while driving, which takes the driver's attention away from the primary task of driving, has increased the number of traffic incidents and crashes (Mokhtarimousavi et al., 2020).

${ }^{1}$ Corresponding author: saaha1@morgan.edu 
According to the NHTSA, about $5.3 \%$ of drivers used either hands-free or hand-held phones while driving at a typical daylight moment in 2017 (NHTSA, 2015).

Driver distraction happens when an object or event attracts a person's attention away from the driving task. Distraction deteriorates driving performance by diverting the driver's eyes from the road (Visual distraction) like adjusting the GPS (Haigney \& Westerman, 2001) or taking the driver's mind off the driving task (cognitive distraction) like conversing on a hands-free cellphone (Recarte \& Nunes, 2003). Besides talking on the phone or to a passenger and interacting with in-vehicle information technologies like a navigation system, other distracting activities are adjusting the radio, online shopping, makeup, eating, and drinking. Several researchers investigated the impact of different types of distraction on driving behavior, including interacting with a phone (Motamedi et al., 2015; Ranney et al., 2005, 2001), reading (text message and email) (Ranney et al., 2001), navigation tasks (Burns et al., 2005; Ranney et al., 2001), interacting with a music player (Ranney et al., 2005, 2001), memory tasks, and classifying sentences (Chaparro et al., 2005; Hurwitz \& Wheatley, 2001). In addition to the type of distraction, the impact of different distracting tasks depends on several other factors such as experience (Chisholm et al., 2008), fatigue (Desmond \& Matthews, 1997), road conditions (e.g., curves, turns, crossings, and amount of traffic) (Ihata et al., 2002), and age (Chaparro et al., 2005; Merat et al., 2005). All the studies mentioned above confirmed the detrimental effect of different types of distraction on driving performance such as lane changing (Blanco et al., 2006; Lansdown et al., 2002), (Burns et al., 2005); reduced anticipation of the need to brake and shortened time to collision (Jamson et al., 2004); and lowered the performance of car-following (Blanco et al., 2006; Lansdown et al., 2004; Ranney et al., 2005).

Driving behavior with and without each distraction should be compared to detect the pure effect of driver distraction. Visual and cognitive distractions represent the two main types of distraction (Liang \& Lee, 2010). A visual distraction that involves the driver's eyes off the road can be determined by temporary changes in drivers' eye glances. Detecting cognitive distraction, in which the mind is off the road, is more complicated than detecting visual distraction because the symptom of mental distraction is generally not apparent and can differ among drivers. Quantifying the complicated relationship between drivers' cognitive states and distraction indicators is a big challenge.

Machine learning and data mining approaches that can extract unknown patterns from a large volume of data demonstrate an advanced and promising method for coping with such a challenge. This skill can mainly be applied to build a discrimination model based on driving behavior without distraction. A Support Vector Machine (SVM), which was proposed in (Vapnik, 2013), is based on the statistical learning method and can be used for pattern classification and implication of nonlinear associations among variables (Cristianini \& Shawe-Taylor, 2000; Vapnik, 2013). This technique has been applied effectively to the recognition and confirmation of faces, objects, handwritten characters and digits, text, speech, and speakers, and the recovery of data and images (Byun \& Lee, 2002; Dehzangi, Paliwal, Lyons et al., 2013; Dehzangi, Paliwal, Sharma et al., 2013). The learning procedure and the strength of the 
SVM for pattern recognition make it an excellent candidate for our problem. SVMs can create linear and nonlinear models and compute the nonlinear models as accurately as the linear ones. The primary advantage of an SVM is its ability to minimize both structural and empirical risk (Khandoker et al., 2009), thereby leading to better generalizations for new data classifications, even with limited training datasets. The SVM can also perform well with noisy data (Byun \& Lee, 2002) and does not require prior knowledge before training. Most of the proposed distraction detection systems are based on supervised learning. Human experts supervise the training by making labels, which are the true distraction states consistent with training data. To yield more strong models than traditional learning methods (e.g., logistic regression), which merely minimize training error, the SVM method avoids overfitting by reducing the upper bound of the simplification error and by finding the maximal marginal hyperplane (Amari \& Wu, 1999). The SVM method is a robust technique for detecting changes in human behavior and represents a particularly promising approach to detecting cognitive distraction given the complex dynamics of eye movement. The SVM is among the most powerful techniques for pattern recognition.

This paper's main objective is to detect and classify driver distraction based on different road types by applying machine learning algorithms (comparing different methods) and using driving simulator data as inputs to the model. Our previous paper (Ahangari et al., 2020) investigated the driving behavior of different distraction types. Here, we mainly address driver distraction on driving behavior (change lane, speed, crash, brake, and throttle) that is considered an essential aspect in the investigated maneuvers.
Different road classes have different speed limits, numbers of lanes, cognitive loads, and traffic loads. Therefore, drivers may act differently on each road type when distracted and may have a higher risk of crashes and near-crashes on some roads than others. To tackle this problem, we used the SVM to our data collected in a simulator study and achieved $94.24 \%$ prediction accuracy for detecting cognitive distraction. This result was obtained on the normalized data, which was tested using ANOVA to validate its statistical significance.

This paper is organized as follows. First, it defines driver distraction based on the current discussion in the literature. Then, the investigated machine learning techniques for modeling driver distraction are briefly described. The experimental setup and the main results are shown next. Finally, the last section summarizes the main points of interest.

\section{Methods}

Two surveys and a driving simulation experiment were conducted to investigate distracted drivers' performance associated with different types of distraction on four different road types (rural collector, freeway, urban arterial, and local road in a school zone). The driving simulation experiment examined an individual's driving performance with different forms of distraction (hands-free calling, hand-held calling, voice command, texting, clothing, and eating/drinking) on different road types. The designed experiment allowed a complete analysis of each participant's driving performance (speed, acceleration, brake, throttle, and crash), given various kinds of roads as well as different forms of distraction. 
Two surveys were designed for this study. The pre-survey, which participants filled out before their driving experience, included sociodemographic information and questions about distracted driving habits (if they use a cellphone when driving, have a crash when using GPS). The post-survey that participants filled out after their driving simulator experience included the probability of using a cellphone or other distracting tasks when driving after being involved in this study. A total of 92 participants, 40 females and 52 males, participated in the survey. Around 60 percent of participants were under 26 years old. The participants' demographic information is shown in our previous study (Ahangari et al., 2020).

We designed the driving simulation experiment to analyze the effect of distraction on four different road types (rural collector, freeway, urban arterial, and local road in a school zone). Participants (ages 18 to 40 ) took part in the experiment. The participants were recruited from Morgan State University and the Baltimore metropolitan area using flyers and online invitations. Some $71.7 \%$ of the participants had their drivers' licenses for at least three years, and most of the participants drive at least 8,000 miles annually. Some 7.6 percent of the participants had crashed while driving distracted. All driving experiments were conducted in the Safety and Behavioral Analysis (SABA) Center at Morgan State University.

We used a high-fidelity driving simulator in the experiment. The simulator's software (Virtual Reality Design Studio) provides a real-world driving atmosphere customized for various applications. It simulates buildings, roads, pavement markings, traffic signals, road signs, weather conditions, and traffic. The driving simulator, produced by the Forum 8 Company, is a fixed driving simulator that consists of a regular vehicle and three 40-inch monitors (FORUM8, 2020). Participants interact with the simulator using a sedan's steering wheel and pedals that provide real-time feedback. Due to the consideration of different types of distraction and four different road conditions, six scenarios were designed for this study. All six scenarios used mild traffic conditions to investigate only the effect of distraction on driving behavior.

The participants engaged in six scenarios, including hands-free calling, hand-held calling, voice command, texting, clothing, and eating/drinking. In each scenario, the participants drove from Hampton Lane (rural road) to I-695 (freeway) to Perring Parkway (urban arterial) to Radar Road (local, school zone), which takes about 15 minutes. The participants were compensated at $\$ 15$ per hour for their participation in the study. They were penalized for disobeying traffic rules such as driving over the speed limit or running a red light. The six scenarios are the same, except for the type of distraction. Participants received one of the six distracting tasks - hands-free calling, hand-held calling, voice command, texting, clothing, and eating/drinking - at the same location in each scenario. The participants used their smartphones to eliminate any discrepancies caused by using an unfamiliar smartphone.

It is worth noting that at the beginning of each scenario, participants were informed about the type of distraction they would receive. Two observers monitored the participants' driving performance. The simulator software recorded all related data, including speed, acceleration, throttle, lane 
changing, brake, collision, steering wheel changing, duration time, and vehicle location every second.

To forecast the distraction based on the driver's behavior on different road types (rural collector, freeway, urban arterial, and local road in a school zone), we normalized the data to have a fair comparison (explained in detail in the next section). We then classified them into four different categories based on the road type. There were 1,952 simulation runs by the 92 participants.

\subsection{Support Vector Machine (SVM)}

A wide range of classification techniques has been used for object detection, such as SVM (Oren et al., 1997), AdaBoost (Rätsch et al., 2001), and neural network (Zhao \& Thorpe, 2000). Some researchers also constructed a classifier of simple cascade features for object detection (Schneiderman, 2004; Viola \& Jones, 2001). Among those classifiers, the SVM is considered one of the best machine learning and pattern classification algorithms and obtained promising results (Dehzangi et al., 2015; Jahangiri \& Rakha, 2015). The SVM finds the maximum marginal hyperplane to minimize classification error. The SVM uses a kernel function to map training data from the original input space to a high-dimensional feature space (Cristianini \& Shawe-Taylor, 2000). When the data are linearly separable in the feature space, the hyperplane that maximizes the margin between classes yields the minimized upper bound of generalization error. However, when the data are not linearly separable, a positive penalty parameter is introduced to permit training error by specifying the cost of misclassifying training instances and find the soft margin. The classification of some known point in input space $\boldsymbol{x}_{i}$ is $y_{i}$ which is defined to be either -1 or +1 . If $\mathbf{x}^{\prime}$ is a point in input space with unknown classification, then (eq.1.):

$y^{\prime}=\operatorname{sign}\left(\sum_{i=1}^{n} \alpha_{i} y_{i} K\left(\mathbf{x}_{i}, \mathbf{x}^{\prime}\right)+b\right)$

where $y^{\prime}$ is the predicted class of point $\mathbf{x}^{\prime}$.

The function $K($ )is the kernel; $n$ is the number of support vectors; $\alpha_{i}$ are adjustable weights, and $b$ is a bias. The SVM training process minimizes both training error and the upper bounds of generalization error.

This method is computationally effective and reduces generalization errors to avoid over-fitting (Dehzangi et al., 2015; Jahangiri \& Rakha, 2015). The SVM yields more robust models in comparison with the linear-regression algorithms that minimize the mean square error, which can be seriously affected by outliers in training data (Dehzangi, Paliwal, Sharma et al., 2013).

\section{Results and Discussion}

\subsection{Statistical Preprocessing using ANOVA}

We used an ANOVA and Post Hoc Tukey to find differences in distracted driving behavior on different road types. Speed, 
throttle (ratio on the acceleration pedal from 0 to +1 in which 0 means no throttle and +1 is full throttle), brake (ratio on the brake pedal from 0 to +1 in which 0 means no braking force and +1 is maximum brake force), steering velocity (rotation rate of the steering wheel per second), offset from road center (offset of the vehicle's position from the center of the road in meters; a negative number shows the offset toward left and a positive number indicates the offset toward the right), lane change, collision, and brake light (frequency of brake lights turning on) are dependent variables. Road type (rural, freeway, urban, and local) is the independent variable.

All variables are only for the distraction period; all but lane changing and brake light (total number during distraction) are the averages over the distracting period for all types of distraction for each road.

Since different road types have different speed limits and numbers of lanes, to have a fair comparison of driving behavior under distraction on different roads, we first normalized data and then performed an ANOVA analysis. The speed limit on the rural road is $30 \mathrm{mph}$, the freeway is 55 , the urban arterial is 45 , and the local road is 30 ; also, the number of lanes in each direction on the rural road is one, the freeway has three, the urban arterial has two, and the local road has one. We subtract the vehicle speed from the speed limit to normalize speed and divide it by the speed limit. For normalizing the lane change, we calculated the number of lanes changing per lane. The ANOVA (Table 1) shows that distracted driving behavior is significantly different under different road types for all variables except collision.

As shown in Table 1, speed on the rural road had the highest ratio to its speed limit under distraction, which could be due to the lower cognitive load of this road type compared to other roads: only one lane in each direction, low traffic, very few intersections and stop signs. Conversely, local roads in a school zone had the lowest ratio to its speed limit under distraction due to higher cognitive load: the presence of pedestrians and frequent stop signs. Drivers generally reduce their speed when distracted (Horrey \& Wickens, 2006); however, our results show that they reduce their speed on local roads in a school zone the most, followed by the urban arterial the freeway. On rural roads, their speed is over the speed limit when distracted. This ranking can be explained by cognitive load. As expected, the number of lane changes is the highest in urban arterials. Also, as expected, steering velocity and offset from the lane center are the highest on the freeway, then the urban road, then rural and, lastly, local road due to their speed limits. Throttle and brake rankings are freeways, rural, urban, and local due to speed and cognitive load. An average number of brake lights rankings are urban arterial, rural, local, and freeway, probably due to a combination of interruptions/cognitive load and speed limit. 


\section{Table 1}

Descriptive and ANOVA Analysis

\begin{tabular}{|c|c|c|c|c|c|c|}
\hline \multicolumn{2}{|c|}{ Variables } & \multirow{2}{*}{$\begin{array}{c}\mathbf{N} \\
402 \\
\end{array}$} & \multirow{2}{*}{$\begin{array}{c}\text { Mean } \\
0.263 \\
\end{array}$} & \multirow{2}{*}{$\begin{array}{c}\begin{array}{c}\text { Std. } \\
\text { Deviation }\end{array} \\
0.314\end{array}$} & \multirow{5}{*}{$\begin{array}{c}\text { F } \\
865.849\end{array}$} & \multirow{5}{*}{$\begin{array}{l}\text { Sig. } \\
0.000\end{array}$} \\
\hline \multirow{4}{*}{ Normalized Speed } & Rural Road & & & & & \\
\hline & Freeway & 800 & -0.128 & 0.088 & & \\
\hline & Urban Arterial & 399 & -0.336 & 0.126 & & \\
\hline & Local & 351 & -0.321 & 0.219 & & \\
\hline \multirow{4}{*}{ Throttle } & Rural Road & 402 & 0.230 & 0.111 & \multirow{4}{*}{287.391} & \multirow{4}{*}{0.000} \\
\hline & Freeway & 800 & 0.243 & 0.094 & & \\
\hline & Urban Arterial & 399 & 0.114 & 0.062 & & \\
\hline & Local & 351 & 0.111 & 0.094 & & \\
\hline \multirow{4}{*}{ Brake } & Rural Road & 402 & 0.041 & 0.048 & \multirow{4}{*}{370.743} & \multirow{4}{*}{0.000} \\
\hline & Freeway & 800 & 0.007 & 0.007 & & \\
\hline & Urban Arterial & 399 & 0.068 & 0.055 & & \\
\hline & Local & 351 & 0.149 & 0.138 & & \\
\hline \multirow{4}{*}{ Steering Velocity } & Rural Road & 402 & 0.012 & 0.009 & \multirow{4}{*}{187.104} & \multirow{4}{*}{0.000} \\
\hline & Freeway & 800 & 0.031 & 0.021 & & \\
\hline & Urban Arterial & 399 & 0.016 & 0.010 & & \\
\hline & Local & 351 & 0.013 & 0.015 & & \\
\hline \multirow{4}{*}{$\begin{array}{l}\text { Offset from Road } \\
\text { Center }\end{array}$} & Rural Road & 402 & 1.077 & 0.640 & \multirow{4}{*}{223.594} & \multirow{4}{*}{0.000} \\
\hline & Freeway & 800 & 3.679 & 2.887 & & \\
\hline & Urban Arterial & 399 & 2.418 & 2.124 & & \\
\hline & Local & 351 & 0.726 & 0.441 & & \\
\hline \multirow{4}{*}{$\begin{array}{c}\text { Normalized Lane } \\
\text { Change }\end{array}$} & Rural Road & 402 & 0.000 & 0.000 & \multirow{4}{*}{380.228} & \multirow{4}{*}{0.000} \\
\hline & Freeway & 800 & 0.507 & 0.549 & & \\
\hline & Urban Arterial & 399 & 0.816 & 0.492 & & \\
\hline & Local & 351 & 0.000 & 0.000 & & \\
\hline \multirow{4}{*}{ Collision } & Rural Road & 402 & 0.000 & 0.000 & \multirow{4}{*}{1.811} & \multirow{4}{*}{0.143} \\
\hline & Freeway & 800 & 0.010 & 0.111 & & \\
\hline & Urban Arterial & 399 & 0.005 & 0.100 & & \\
\hline & Local & 351 & 0.000 & 0.000 & & \\
\hline \multirow{4}{*}{ Brake Light } & Rural Road & 402 & 1.264 & 2.414 & \multirow{4}{*}{87.713} & \multirow{4}{*}{0.000} \\
\hline & Freeway & 800 & 0.369 & 1.150 & & \\
\hline & Urban Arterial & 399 & 3.291 & 5.395 & & \\
\hline & Local & 351 & 1.222 & 2.319 & & \\
\hline
\end{tabular}

Tukey Post Hoc analysis, as shown in brake, steering velocity, offset from road Table 2, reveals a statistically significant center, lane change, and brake light among difference in the mean of speed, throttle, different road types. 
Table 2

Tukey's Post Hoc Analysis of Distracted Driving Behavior

\begin{tabular}{|c|c|c|c|c|c|}
\hline \multicolumn{3}{|c|}{ Variables } & \multirow{2}{*}{$\begin{array}{c}\text { Mean Difference } \\
(\mathbf{I}-\mathbf{J})\end{array}$} & \multirow{2}{*}{$\begin{array}{c}\text { Std. Error } \\
0.01149 \\
\end{array}$} & \multirow{2}{*}{$\begin{array}{l}\text { Sig. } \\
0.000 \\
\end{array}$} \\
\hline \multirow{3}{*}{ Normalized Speed } & \multirow{3}{*}{ Rural Road } & Freeway & & & \\
\hline & & Urban Arterial & $0.59923^{*}$ & 0.01328 & 0.000 \\
\hline & & Local & $0.58342 *$ & 0.01373 & 0.000 \\
\hline \multirow{3}{*}{ Throttle } & \multirow{3}{*}{ Rural Road } & Freeway & -0.01374 & 0.00567 & 0.073 \\
\hline & & Urban Arterial & $.11520^{*}$ & 0.00655 & 0.000 \\
\hline & & Local & $.11909^{*}$ & 0.00677 & 0.000 \\
\hline \multirow{3}{*}{ Brake } & \multirow{3}{*}{ Rural Road } & Freeway & $.03421^{*}$ & 0.00412 & 0.000 \\
\hline & & Urban Arterial & $-.02647^{*}$ & 0.00476 & 0.000 \\
\hline & & Local & $-.10763^{*}$ & 0.00493 & 0.000 \\
\hline \multirow{3}{*}{ Steering Velocity } & \multirow{3}{*}{ Rural Road } & Freeway & $-.01881^{*}$ & 0.00098 & 0.000 \\
\hline & & Urban Arterial & $-.00354^{*}$ & 0.00113 & 0.010 \\
\hline & & Local & -0.00088 & 0.00117 & 0.876 \\
\hline \multirow{3}{*}{$\begin{array}{l}\text { Offset from Road } \\
\text { Center }\end{array}$} & \multirow{3}{*}{ Rural Road } & Freeway & $-2.60195^{*}$ & 0.12912 & 0.000 \\
\hline & & Urban Arterial & $-1.34103^{*}$ & 0.14925 & 0.000 \\
\hline & & Local & 0.35134 & 0.15429 & 0.104 \\
\hline \multirow{3}{*}{$\begin{array}{c}\text { Normalized Lane } \\
\text { Change }\end{array}$} & \multirow{3}{*}{ Rural Road } & Freeway & $-0.50708^{*}$ & 0.02543 & 0.000 \\
\hline & & Urban Arterial & $-0.81578^{*}$ & 0.02940 & 0.000 \\
\hline & & Local & 0.00000 & 0.03039 & 1.000 \\
\hline \multirow{3}{*}{ Collision } & \multirow{3}{*}{ Rural Road } & Freeway & -0.01000 & 0.00517 & 0.213 \\
\hline & & Urban Arterial & -0.00501 & 0.00597 & 0.836 \\
\hline & & Local & 0.00000 & 0.00617 & 1.000 \\
\hline \multirow{3}{*}{ Brake Light } & \multirow{3}{*}{ Rural Road } & Freeway & $.89493^{*}$ & 0.17986 & 0.000 \\
\hline & & Urban Arterial & $-2.02705^{*}$ & 0.20791 & 0.000 \\
\hline & & Local & 0.04146 & 0.21492 & 0.997 \\
\hline
\end{tabular}

Note: *. The mean difference is significant at the 0.05 level.

\subsection{Classification Results}

To evaluate our model, we randomly split our data for each subject set (1,952 experiments) into training ( $80 \%$ of the samples) and independent test sets ( $20 \%$ of the samples). As a result, we have 1,587 samples in our training data set and 365 samples in our testing data set. We then report our result for 10 -fold cross-validation on the training data and the results on the independent test set. In 10 -fold cross-validation, the data is randomly divided into ten equal subgroups. In each iteration, nine subgroups are combined as training data, and the remaining subset is used as the testing data. This process is repeated ten times, equal to the number of subgroups. Data were sampled at random from the training set, as all data used in this study were labeled adequately as distracted under four different road conditions. For a fair comparison, the random data partition was conducted once and saved, and different algorithms were run over the same classification. Note that the independent test set has never been used for parameter tuning to investigate our achieved results' generality. 
We classify four different distraction definitions based on the road types (rural collector, freeway, urban arterial, and local road in a school zone) using SVMs. To provide more insight into the performance of the SVM, we report Sensitivity, Precision, Matthew Correlation Coefficient (MCC), Area Under the Curve (AUC), and total Accuracy (ACC). Our achieved results using 10 -fold cross-validation and an independent test set are shown in Table 4.

As shown in Table 3, the SVM can predict the road's distraction with $94.24 \%$ accuracy for the independent test set and $93.90 \%$ for 10 -fold cross-validation. The similar results achieved for these two evaluation methods demonstrate the generality of our achieved results. As shown in Table 3 , we can predict the distraction for the freeway, urban arterial, and local road in a school zone with over $90 \%$ specificity. This demonstrates the effectiveness of the SVM to predict distraction in such roads. The mean sensitivity for all SVM models is $92.75 \%$. The high Sensitivity and Precision demonstrate the SVM's ability to predict positive samples. In other words, they demonstrate that the SVM can identify different distraction patterns with respect to varying roads with more than $90 \%$ accuracy.

This study uses machine learning to predict different distraction patterns for various driving environments. Using proper preprocessing to normalize our data and validating that using ANOVA enables us to make our data consistent for further analysis. After that, using the SVM as our classification technique, we can achieve $94.24 \%$ accuracy for this task, which is a promising result. Such a study and produced data can be used as a benchmark for future studies in this field.

\section{Table 3}

Results Achieved Using SVM for 10-fold Cross-validation and Independent Test Set

\begin{tabular}{|c|c|c|c|c|c|}
\hline \multicolumn{6}{|c|}{ Results of 10-fold cross-validation } \\
\hline & Sensitivity & Precision & MCC & AUC & ACC \\
\hline Rural Collector & $92.30 \%$ & $92.30 \%$ & $90.30 \%$ & $97.20 \%$ & --- \\
\hline Freeway & $97.80 \%$ & $93.30 \%$ & $92.30 \%$ & $96.60 \%$ & --- \\
\hline Urban arterial & $94.00 \%$ & $98.20 \%$ & $95.10 \%$ & $98.40 \%$ & --- \\
\hline Local road in a School Zone & $86.90 \%$ & $92.40 \%$ & $87.40 \%$ & $96.50 \%$ & --- \\
\hline Total & & & & & $93.90 \%$ \\
\hline \multicolumn{6}{|c|}{ Results of Independent Test } \\
\hline & Sensitivity & Precision & MCC & AUC & ACC \\
\hline Rural Collector & $93.40 \%$ & $98.60 \%$ & $95.00 \%$ & $98.40 \%$ & --- \\
\hline Freeway & $99.30 \%$ & $89.80 \%$ & $90.30 \%$ & $95.90 \%$ & --- \\
\hline Urban arterial & $92.00 \%$ & $98.60 \%$ & $94.10 \%$ & $98.30 \%$ & --- \\
\hline Local road in a School Zone & $85.90 \%$ & $96.50 \%$ & $89.30 \%$ & $94.40 \%$ & --- \\
\hline Total & & & & & $94.24 \%$ \\
\hline
\end{tabular}

\section{Conclusions}

In this paper, we recruited 92 participants to drive on a simulated network in the Baltimore area (Maryland, USA) - including a rural road, a freeway, an urban arterial, and a local road in a school zone - under six different driving distractions in a highfidelity driving simulator. Their driving performances were recorded and analyzed. 
The ANOVA and Tukey Post Hoc results indicated that participants demonstrate different driving behavior under distraction on different road types. However, the number of crashes is not significantly different on various roads. The Support Vector Machine (SVM) method recognized and predicted the pattern with $94.24 \%$ accuracy for the independent test set and $93.9 \%$ for 10 -fold cross-validation.

The results showed that the participants drove over the speed limit when distracted on rural roads. This is most probably due to very low traffic flow and low cognitive load, which could increase the probability of crashes in an interruption such as an animal passing. Conversely, driving on freeways at $13 \%$ under the speed limit (to focus on the distracting event such as texting) could cause crashes with cars moving at speeds higher than the speed limit, especially in the left lanes. Driving on the freeway had the least force of brake pedal while driving on the local road had the most, and that can be related to a load of scenery and intersections. On all four different roads, there was an offset from the road center toward the right; the freeway had the most offset, and the local road had the least.

This study is the first of its kind to the best of our knowledge using machine learning to predict different distraction patterns for the various roads and driving environments. Our previous study (Ahangari et al., 2020) reported the effect of different types of distractions, and this study presented the distracted model under different road classes. A limitation of this study is that it investigated various distractions combined with driving behavior on different roads. An extension of this study would be collecting more data and classifying them by road and distraction type. Furthermore, comparing driving behavior when distracted and not distracted on each road type would help model distracted behavior. The extended prediction model could be used by law enforcement agencies and auto insurance companies to recognize driver distraction type using recorded vehicle performance measures such as speed, steering velocity, brake, etc., on different roads.

\section{Acknowledgments}

The authors would like to thank the Maryland Department of TransportationMotor Vehicle Administration-Maryland Highway Safety Office (GN-Morgan State-2019-291) and the Urban Mobility \& Equity Center at Morgan State University for their funding support.

\section{References}

Ahangari, S.; Chavis, C.; Jeihani, M.; Moghaddam, Z. R. 2018. Quantifying the Effect of On-Street Parking Information on Congestion Mitigation using a Driving Simulator, Transportation Research Record, 2672(8): 920929.

Ahangari, S.; Jeihani, M.; Salahshour, B.; Ndegwa, M. 2020. A Comprehensive Analysis of Distracted Driving Using a Driving Simulator, International Journal for Traffic \& Transport Engineering 10(2): 252-265.

Ahangari, S.; Rashidi Moghaddam, Z.; Jeihani, M.; Chavis, C.; Chen, H.; Rakha, H.; Kang, K. 2019. Investigating the Effectiveness of an Eco-Speed Control System in the Vicinity of Signalized Intersections Using a Driving Simulator. Transportation Research Board 98th Annual Meeting, Washington DC, United States. 20 p.

Amari, S.; Wu, S. 1999. Improving support vector machine classifiers by modifying kernel functions, Neural Networks 12(6): 783-789. 
Blanco, M.; Biever, W.J.; Gallagher, J. P.; Dingus, T. A. 2006. The impact of secondary task cognitive processing demand on driving performance, Accident Analysis \& Prevention 38(5): 895-906.

Burns, P. C.; Trbovich, P. L.; McCurdie, T.; Harbluk, J. L. 2005. Measuring distraction: Task duration and the lane-change test (LCT). In Proceedings of the Human Factors and Ergonomics Society Annual Meeting, 49: 19801983.

Byun, H.; Lee, S.-W. 2002. Applications of support vector machines for pattern recognition: A survey. International Workshop on Support Vector Machines, 213236.

Chaparro, A.; Wood, J. M.; Carberry, T. 2005. Effects of age and auditory and visual dual tasks on closedroad driving performance, Optometry and Vision Science 82(8): 747-754.

Chisholm, S. L.; Caird, J. K.; Lockhart, J. 2008. The effects of practice with MP3 players on driving performance, Accident Analysis \& Prevention 40(2): 704-713.

Cristianini, N.; Shawe-Taylor, J. 2000. An introduction to support vector machines and other kernel-based learning methods. Cambridge university press. $189 \mathrm{p}$.

Dehzangi, A.; Heffernan, R.; Sharma, A.; Lyons, J.; Paliwal, K.; Sattar, A. 2015. Gram-positive and Gram-negative protein subcellular localization by incorporating evolutionary-based descriptors into Chou $\mathrm{s}$ general PseAAC, Journal of Theoretical Biology 364: 284-294.

Dehzangi, A.; Paliwal, K.; Lyons, J.; Sharma, A.; Sattar, A. 2013. Enhancing protein fold prediction accuracy using evolutionary and structural features. In IAPR International Conference on Pattern Recognition in Bioinformatics, 196-207.
Dehzangi, A.; Paliwal, K.; Sharma, A.; Lyons, J.; Sattar, A. 2013. Protein fold recognition using an overlapping segmentation approach and a mixture of feature extraction models. In Australasian Joint Conference on Artificial Intelligence, 32-43.

Desmond, P. A.; Matthews, G. 1997. Implications of taskinduced fatigue effects for in-vehicle countermeasures to driver fatigue, Accident Analysis \& Prevention 29(4): 515-523.

Ebnali, M.; Lamb, R.; Fathi, R. 2020. Familiarization tours for first-time users of highly automated cars: Comparing the effects of virtual environments with different levels of interaction fidelity, Journal of Applied Ergonomics (in print).

FORUM8. 2020. VR software, BIM solution, civil engineering design software, structural analysis software, simulation. (n.d.). Available from Internet: <https://www.forum8.co.jp/index.html>. [Retrieved July 23, 2020].

Haigney, D.; Westerman, S. J. 2001. Mobile (cellular) phone use and driving: A critical review of research methodology, Ergonomics 44(2): 132-143.

Horrey, W. J.; Wickens, C. D. 2006. Examining the impact of cell phone conversations on driving using meta-analytic techniques, Human Factors 48(1): 196205.

Hurwitz, J. B.; Wheatley, D. J. 2001. Driver choice of headway with auditory warnings. In Proceedings of the Human Factors and Ergonomics Society Annual Meeting, 45: 1637-1640.

Ihata, N.; Ikegami, M.; Kawaguchi, M.; Hasegawa, H.; Ayama, M.; Kasuga, M. 2002. Reaction time of the secondary task while driving in various situations. In Proceedings of the IEEE 5th International Conference on Intelligent Transportation Systems, 274-278. 
Jahangiri, A.; Rakha, H. A. 2015. Applying machine learning techniques to transportation mode recognition using mobile phone sensor data, IEEE Transactions on Intelligent Transportation Systems 16(5): 2406-2417.

Jamson, A. H.; Westerman, S. J.; Hockey, G. R. J.; Carsten, O. M. 2004. Speech-based email and driver behavior: Effects of an in-vehicle message system interface, Human Factors 46(4): 625-639.

Khandoker, A. H.; Gubbi, J.; Palaniswami, M. 2009. Automated scoring of obstructive sleep apnea and hypopnea events using short-term electrocardiogram recordings, IEEE Transactions on Information Technology in Biomedicine 13(6): 1057-1067.

Lansdown, T. C.; Brook-Carter, N.; Kersloot, T. 2002. Primary task disruption from multiple in-vehicle systems, ITS Journal-Intelligent Transportation Systems Journal 7(2): 151-168.

Lansdown, T. C.; Brook-Carter, N.; \& Kersloot, T. 2004. Distraction from multiple in-vehicle secondary tasks: Vehicle performance and mental workload implications, Ergonomics 47(1): 91-104.

Lee, J. D.; Caven, B.; Haake, S.; Brown, T. L. 2001. Speech-based interaction with in-vehicle computers: The effect of speech-based email on drivers' attention to the roadway, Human Factors 43(4): 631-640.

Liang, Y.; Lee, J. D. 2010. Combining cognitive and visual distraction: Less than the sum of its parts, Accident Analysis \& Prevention 42(3): 881-890.

Merat, N.; Anttila, V.; Luoma, J. 2005. Comparing the driving performance of average and older drivers: The effect of surrogate in-vehicle information systems, Transportation Research Part F: Traffic Psychology and Behaviour 8(2): 147-166.
Mokhtarimousavi, S.; Anderson, J. C.; Azizinamini, A.; Hadi, M. 2020. Factors affecting injury severity in vehicle-pedestrian crashes: A day-of-week analysis using random parameter ordered response models and Artificial Neural Networks, International Journal of Transportation Science and Technology 9(2): 100-115.

Motamedi, S.; Hasheminejad, M.; Choe, P. 2015. Driving safety considered user interface of a smartphone: An experimental comparison, International Conference on Cross-Cultural Design, 150-160.

NHTSA. 2015. Welcome to ROSA P | National Survey on Distracted Driving Attitudes and Behaviors - 2015 - 35960 | NHTSA - Behavioral Safety Research. Available from Internet: <https://rosap.ntl.bts.gov/view/dot/35960>.

Oren, M.; Papageorigiou, C.; Sinha, P.; Osuna, E.; Poggio, T. 1997. Pedestrian detection using wavelet templates. In Proceedings of IEEE computer society Conference on computer vision and pattern recognition, 193-199.

Ranney, T. A.; Harbluk, J. L.; Noy, Y. I. 2005. Effects of voice technology on test track driving performance: Implications for driver distraction, Human Factors 47(2): 439-454.

Ranney, T. A.; Mazzae, E. N.; Garrott, W. R.; Barickman, F. S. 2001. Development of a test protocol to demonstrate the effects of secondary tasks on closed-course driving performance. In Proceedings of the Human Factors and Ergonomics Society Annual Meeting, 45: 1581-1585.

Rätsch, G.; Onoda, T.; Müller, K.-R. 2001. Soft margins for AdaBoost, Machine Learning 42(3): 287-320.

Recarte, M. A.; Nunes, L. M. 2003. Mental workload while driving: Effects on visual search, discrimination, and decision making, Journal of Experimental Psychology: Applied 9(2): 119-137. 
Schneiderman, H. 2004. Feature-centric evaluation for efficient cascaded object detection. In Proceedings of the 2004 IEEE Computer Society Conference on Computer Vision and Pattern Recognition, CVPR 2004, 2(4): 29-36.

Strayer, D. L.; Drews, F. A.; Johnston, W. A. 2003. Cell phone-induced failures of visual attention during simulated driving, Journal of Experimental Psychology: Applied 9(1): 23-32.

Vapnik, V. 2013. The nature of statistical learning theory. Springer Heidelberg. 314 p.
Viola, P., \& Jones, M. (2001). Rapid object detection using a boosted cascade of simple features. In Proceedings of the 2001 IEEE computer society conference on computer vision and pattern recognition, CVPR 2001, 1(1): 511-518.

Zhao, L.; Thorpe, C. 2000. Stereo-and neural networkbased pedestrian detection, IEEE Transactions on intelligent transportation systems 1(3): 148-154.

\section{jitte 114}

